\section{Der medizinische Anteil an der Gründung der Berliner Universität.}

\section{Von J. Schwalbe.}

In volltönenden Akkorden werden die Hymnen zu Ehr und Preis der Universitas Friderico-Guilelma Berolinensis am Ende ihres ersten Säkulum erschallen. Unter allen Ruhmesworten kann keines höher gewertet werden, keines mehr unseren Stolz auf die Berliner Alma mater rechtfertigen, als der Hinweis, daß ihre Entwicklung mit dem Wachstum des engeren und weiteren Vaterlandes innig verknüpft gewesen ist, daß ihr Aufstieg ein leuchtendes Sinnbild für die glorreiche Geschichte Preußens und Deutschlands bedeutet, ja, daß ihr Leben und Wirken selbst zu dem Aufblühen unserer Nation in nicht geringem $\mathrm{Maße}$ die ununterbrochene Triebkraft geliefert hat.

Als die Berliner Universität gegründet wurde, lag Preußen geknechtet am Boden, unter dein eisernen Druck des französischen Weltherrschers - und jetzt, wo das erste Jahrhundert ihres Daseins sich vollendet, ist sie ein Edelstein in der Krone des geeinten deutschen Vaterlandes, das im Rate der Völker keinem anderen den Vorrang einzuräumen gehalten ist. Das Wort, mit dem König Friedrich Wilhelm III. das Gesuch, an Stelle der fast vernichteten Hallenser Universität eine neue in Berlin zu erbauen, billigte: ,Der Staat muß durch geistige Kräfte ersetzen, was er an physischen verloren hat," ist über alles Erwarten glänzend in Erfüllung gegangen.

Von den herrlichen Früchten, die sich aus den mit vollen Händen ausgestreuten Saaten entwickelt haben, darf die Medizinische Fakultät wahrlich nicht den geringsten Teil für sich in Anspruch nehmen. Die Leistungen ihrer Mitglieder waren fast $\mathrm{zu}$ allen Zeiten denen der Schwester-Fakultäten ebenbürtig, - eine große Fülle von Arbeiten ist aus ihren Reihen hervorgegangen, die die Richtung der wissenschaftlichen Medizin nachhaltig beeinflußt haben, auf dem Boden der Berliner medizinischen Fakultät sind Werke entstanden, die als hoch emporragende Marksteine in der Geschichte der Heilkunde einen dauernden Platz erworben haben, in unserer Fakultät haben Rudolph Virchow und Robert Koch gewirkt, von denen jeder für die medizinische Wissenschaft ein neues Zeitalter geschaffen hat.

Aber nicht nur in der Entwicklungsgeschichte der Berliner Universität hat die Medizin fort und fort einen vornehmen Rang behauptet, auch an ihrer Begründung selbst hat sie einen nicht unwesentlichen Anteil genommen.

Lange vor der Geburt der Universität haben in Berlin medizinische Einrichtungen bestanden, die in einer für die damalige Zeit recht bemerkenswerten Weise der Ausbildung von Heilbeflissenen dienten. Schon im Jahre 1710 war die Charité entstanden - ihre Geschichte ist gelegentlich ihres 200 jährigen $J$ ubiläums in dieser Wochenschrift eingehend geschildert -, wenige Jahre später war das Theatrum anatomicum begründet, das 1724 zum Collegium medico-chirurgicum ausgebaut wurde, und 1795 endlich war das Friedrich Wilhelms-Institut, die Pepinière, gestiftet worden. Alle drei Institute dienten anfänglich lediglich der Ausbildung von Militärärzten und Feldchirurgen, allmählich aber erweiterten sich ihre Aufgaben zum Unterricht für Zivilärzte, ja am Ausgange des 18. und zu Beginn des 19. Jahrhunderts fanden sich die an den Anstalten wirkenden Lehrer bereit, auch öffentliche Vorlesungen $\mathrm{zu}$ halten, an denen das Laienpublikum teilnehmen konnte. Wenn wir den Lektionskatalog des Collegium medico-chirurgicum für das Winterhalbjahr 1806/1807, veröffentlicht in der Spenerschen Zeitung vom 4. Oktober 1806, vergleichen, so finden wir, daß er im wesentlichen alles enthält, was eine kleine medizinische Fakultät ihren Studenten für eine geeignete Ausbildung zu bieten hat.

Es kündigten an:

Hufeland: vier Stunden wöchentlich eine gedrängte Uebersicht seines Systems der praktischen Heilkunde und klinische Uebungen in der Charité. Sprögel: Physiologie nach Anleitung des Herrn v. Hallers
Grundriß. J. G. Walter (öffentlich): die Sinnenorgane und die Nervenlehre, (privatim): Uebungen in der Anatomie und Physiologie und die theoretischen und praktischen Accoucheur.Wissenschaften. Fritze: Klinischer Unterricht in der Charité. Knape (öffentlich): Syndesmologie, (privatim): die praktische Zergliederungskunst, auch, wenn es verlangt wird, die Staats-Arzneiwissenschaft, Anleitung zu gerichtlichen Obduktionen und zu allen übrigen medizinisch-gerichtlichen Untersuchungen. Mursinna (öffentlich): Cursum operationum chirurgicum, (privatim): die Chirurgie, Luxationen, Frakturen-Bandagen, Cursum operationum chirurgicum, auch den praktischen Teil der Geburtshilfe in der Charité. Zenker: Chirurgia medica und Bandagen-Lehre und Operationskursus. F. A. Walter: Muskel-, Blutgefäß- und EingeweideLehre, praktische Zergliederungskunst. Präparate aus dem Gebiet der theoretischen und praktischen Anatomie, Medizin und Chirurgie. Her mbstädt: Allgemeine Grundsätze der medizin isch-praktischen Chemie mit Anwendung auf Zubereitung, Kenntnis und Prüfung der Arzneimittel. Ribke: Geburtshilfe. (Unterricht für Hebammen.) Formey: Erkenntnis und Kur der chronischen Krankheiten. Wildenow: Zoologie, Materia medica. Kiesewetter (Prof. d. Philosophie): Logik, Erfahrungs-Seelenlehre und Moral, reine Mathematik. v. Könen: Materia medica, Grundriß der Physiologie. Augustin: Kriegs.Heilkunde, Physiologie und gesamte Therapie. Grapengiesser: Venerische Krankheiten, Chirurgia medica, Augenkrankheiten, Medizinisch-chirurgisches Clinicum. Hecker: Pathologie, Semiotik, Therapie, Chirurgia medica, Venerische Krankheiten und Gifte. Bourguet (Prof. e. o.): Experimental-Chemie, Experimental-Pharmacie und Experimental-Physik.

Die öffentlichen Vorlesungen waren obligatorisch, die privaten wurden gegen Honorar gelesen. Das unter dem „Königlich preußischen Medizinal-Departement" stehende Collegium hatte einen ständigen Direktor und einen alljährlich unter den ordentlichen Professoren wechselnden Dekan. Die Studenten wurden immatrikuliert. Akademische Würden wurden nicht verliehen.

Gerade das Vorhandensein von zweckmäßigen medizinischen Instituten und Unterrichtsgelegenheiten dürfte manchem der damaligen Zeitgenossen, die auf eine Erweiterung des preußischen Universitätenkreises bedacht waren, den Gedanken nahe gelegt haben, daß Berlin für eine neue akademische Pflanzstätte prädestiniert sei, daß auf der Basis seiner medizinischen Einrichtungen eine neue Universität geschaffen werden könne. $\left.{ }^{1}\right)$ Bemerkenswert ist, daß unter denjenigen Veröffentlichungen, die für die Schöpfung einer Berliner Universität mit Nachıdruck eintraten, die gedankenvolle Schrift eines Berliner ,ausübenden" Arztes besondere Aufmerksamkeit verdient. J. B. Erhard setzt in seiner 1802 erschienenen Monographie „Ueber die Einrichtung und den Zweck der höheren Lehranstalten" seine Ansichten über die Aufgaben und Organisation von Universitäten eingehend auseinander.

Wie er in seiner Vorrede bemerkte, war seine Schrift schon seit vier Jahren fertig. "Ich erinnere dieses, weil seitdem Kants Schrift über den Streit der Fakultäten ${ }^{2}$ ) erschienen ist und der Leser glauben könnte, daß ich daraus meine Ansicht der Fakultäten, wie sie bestehen, genommen hätte, indem ich mit den Grundideen dieser Schrift durchaus einig bin. Es konnte dies aber, da ich mit Kant über die letzten Gründe unserer Erkenntnis gänzlich einverstanden bin, bei konsequentem Denken nicht anders seyn."

Erhard plädiert dafür, daß die Zahl der preußischen Universitäten verringert, daß nur Halle und Königsberg beibehalten und da $\beta$ aus bedeutenden Mitteln Berlins eine neue Universität errichtet werde. Für diese ist der Grundriß bestimmt, den er in seinem ,Entwurf einer vollständigen und z.weckmäßigen Universität" skizziert. Anstatt der bisherigen vier Fakultäten werden unter Ausschaltung der theologischen nur drei gefordert, nämlich die der Philosophie und freien Künste, der Wohlfahrtskunde, welche alles umfaßt, was sich auf Staatsverwaltung bezieht, und der Heilkunde. Bei der letztgenannten sind folgende Lehr. kräfte notwendig: ,,1. Ein Prosector, der auch bei den Versuchen mit lebendigen Thieren Hand leistet. 2. Ein Lehrer der Anatomie, der auch Zootomie lehrt. 3. Der pharmacevtischen Naturgeschichte, der den botanischen Garten und das Naturalienkabinett zuglcich unter sich hat. 4. Der pharmacevtischen Theorie und Recepturkunst, der das Laboratorium unter sich hat. 5. Der Physiologie der organischen Natur, der medicinischen Anthropologie und der Pathologie. 6. Der Pathologie und Therapie in Verbindung, der in seinen Vorlesungen Rücksicht auf das Spital nimmt und Chirurgie theoretisch lehrt. 7. Der Encyklopädie der Heilkunde und der Geschichte derselben. 8. Ein Lehrer der Thierarzneikunde und Viehzucht: 9. des Ackerbaues; 10. der Forstkunde.

1) Dieselben Tendenzen zeigen sich jetzt in Hamburg und Frankfurt a. M

2) Vgl. die folgende Abhandlung von W. A. Freund. 
11. Ein Spital-Arzt, der an Krankenbette Unterricht ertheilt. 12. Ein Spital-Chirurg, der am Krankenbette Vorlesungen hält und den Kursus der Operationen durchmacht. 13. Ein Accoucheur, der praktisch im Accoucheurhause unterrichtet."

Die sonderbare Erweiterung des medizinischen Unterrichts durch denjenigen in der Veterinär-, Forst- und Ackerbaukunde begründet er in folgendem Satze: „Da das Princip der organischen Natur durchaus das nämliche ist, so gehört auch die Behandlung der Pflanzen zun Zweck ihres Wachsthums zur Heilkunde im ganzen Umfange, und wegen der Wichtigkeit' des Ackerbaues für den Staat muß diese vorzüglich gelehrt werden."i)

Bedeutungsvoller als diese allgemeinen Gedanken und Betrachtungen waren die Versuche zur Errichtung einer Berliner Universität, die unter der tatkräftigen Aegide des ausgezeichneten Geheimen Kabinettsrats Beyme in festere Bahnen gelenkt wurden. Auch in dem auf seine Veranlassung im Jahre 1802 ausgearbeiteten Gutachten des Prof. Engel, das den ersten bestimmten Bauplan für eine Berliner Universität enthält, finden wir ebenfalls den oben schon erwähnten Hinweis auf die Bedeutung der Grundlagen, die die in der Hauptstadt bereits existierenden medizinischen Einrichtungen für die Begründung nicht nur einer Medizinischen Fakultät, sondern auch einer Universität gewähren.

„Wo wäre ein so stark besetztes Krankenhaus, ein so reichlich versorgtes anatomisches Theater, soviel Gelegenheit, wirklichen Entbindungen, Krankenbehandlungen, Operationen aller Art beizuwohnen, als in Berlin?" „Die ganze eigentlich kostbare medizinische Fakultät. . ist nach allen Fächern da, und ist in solchem Grade der Volıständigkeit und der Güte da, daß nur wenig zu wünschen übrig seyn kann.

Nicht so weitgehend, sondern nur auf einen Ausbau des Collegium medico-chirurgicum gerichtet waren die amtlichen Gutachten, die von Hufeland, der 1800 von Jena als Direktor des Collegiums nach Berlin berufen war, und von dem Hallenser Kliniker Reil im Jahre 1802 erstattet wurden. Trotzdem können ihre sorgsam ausgearbeiteten Vorschläge als wichtige Beiträge zur Organisation einer Berliner Universität und insbesondere des medizinischen Unterrichts an ihr angesehen werden, und wenigstens das Gutachten $\mathrm{Hufelands}$ verdient sowohl unter diesem Gesichtswinkel als auch wegen seiner allgemeinen Bedeutung für die Geschichte der Medizin an dieser Stelle eine ausführliche Wiedergabe. ${ }^{2}$ )

"Ideen über die Einrichtung des medizinischen Studiums.

Zur Bildung eines brauchbaren und nicht bloß handwerksmäßigen Arztes und Wundarztes gehört:

1) Sehr interessant ist die Polemik Erhards gegen die Verwendung der lateinischen Sprache für wissenschaftliche Zwecke und sein energisches Eintreten für ihren Ersatz durch die deutsche.

,Alle Reden und jetzt bei feierlichen Gelegenheiten herauskommende Schriften müssen, sofern sie nicht unmittelbar die Fertigkeit des Verfassers in einer fremden Sprache beweisen sollen, in der Landessprache seyn, denn die Universität ist zum Wohl des Staates und der Bürger vorhanden, und diese müssen daher an allem Antheil nehmen können.

So nützlich das Studium der gebildeten todten Sprachen ist, so wenig sind sie zur Verbreitung der Wissenschaften nothwendig, denn sie müssen, um neuere Begriffe in ihnen auszudrücken, erweitert werden und da diese Erweiterung selten von jemanden geschieht, der mit dem Genius der Sprache und der Wissenschaft gleich vertraut ist, so werden sie dadurch verdorben. Unser jetziger wissenschaftlicher Vortrag in den philosophischen, juristischen und medicinischen Wissenschaften würde wohl keinem gebildeten Römer verständlich seyn. Die alten Sprachen sind wichtig wegen der Erweiterung der Denkart und der Bildung des ästhetischen Gefühls, das die in ihnen geschriebenen Schriften gewähren, aber sie sind ganz entbehrlich, um unsere Denkart und Ansicht der Dinge in sie hineinzuzwingen

Um die Verbindung der Gelehrten unter sich zu erleichtern, sind sie auch nicht nöthig, denn dazu sind ein paar lebendige Sprachen immer hinlänglich, auch können sie, wenn man ihnen Begriffe und Ansichten der Dinge aufdrängt, welche die Alten nicht hatten, sehr leicht Mißverständnisse hervorbringen. Sie zu den akademischen Ausarbeitungen zu nehmen, um die Studierenden zu zwingen, sich auf selbige zu legen, hat den Nachtheil, daß erstens dem Gesetze durch eine sehr oberflächliche Kenntnis leicht Genüge grthan Werden kann, und dass zweitens dem, der keine Lust daran findet, es zu einer Fertigkeit im sogenannten lateinischen Sprechen und Schreiben zu bringen, viel Zeit geraubt wird, die er weit besser zum Dienste der Wissenschaften hätte verwenden können." - Erst in unseren Tagen ist es nach vielen Mühen gelungen, die lateinische Doktorpromotion abzuschaffen!

2) Für die Erlaubnis zur Veröffentlichung bin ich der Direktion des Geheimen Staatsarchivs zu Dank verpflichtet.
1. daß er die allgemeine Bildung eines Gelehrten erhalte, Humaniora, Geschichte und besonders Sprachunterricht, welches alles auf Schulen geschehen $\mathrm{muB}$;

2. daß er die zu seiner Kunst nötigen Wissenschaften und Kenntnisse sein eigen mache (theoretischen Unterricht in der Medizin). Dazu gehört Philosophie (aber vernünftige), die sich nicht über allcs Wissen erhebt, sondern sich demselben anschmiegt, Mathematik, Physik, Naturgeschichte, Botanik, Chemie, Anatomie, Physiologie, Pathologie, Arzneimittellehre, Therapie, sowohl allgemeine als besondere, Chirurgie, Entbindungskunst;

3. daß er die vollständig erlernten medizinischen Kenntnisse auch zur Heilung am Krankenbette anwenden und rationell handeln lerne. Dies ist nun eine ganz andere Art von Unterricht und Bildung als die vorige. Bei jenem bedarf es weiter nichts, und der Liehrer hat auf weiter nichts zu sehen, als daß der Lehrling das Vorgetragene gut erlerne. Hier aber kommt es darauf an, daß er handele und die mannigfaltigen theoretischen Kenntnisse in Anwendung bringe. Man kann die ganze Medizin wissen, ohne deswegen ein Arzt zu sein. Das Schwerste ist dieser Uebergang vom Wissen zum Handeln, und der Mangel dieser Anleitung war es, der ehedem die jungen Aerzte nötigte, ihn, sich selbst überlassen, zum großen Nachteil ihrer Kranken, in dem ersten Jahr der Praxis zu machen. Diesen Uebergang unter gehöriger Aufsicht zu machen, ist nun der Zweck der Klinik, die ich deswegen für das wichtigste Collegium von allen halte, wo die ganze Masse der medizinischen Kenntnisse erst Einheit und Zweck erhalten und der junge Arzt zum ersten Male seine Kräfte selbst brauchen und kennen lernen soll. Aber eben deswegen ist hier eine ganz eigene Art von Behandlung nötig, und der Lehrer, der dies Collegium wie jedes andere behandelt, $d$. $h$. bloß Vorträge und Demonstrationen, sei es auch am Kranken bette, hält, würde ein schlechter Lehrer der Klinik sein. Ich kann hier aus Erfahrung sprechen, da ich dies Lehramt in Jena selbst acht Jahre lang verwaltet habe. Hier muß nämlich der Lehrer sich mehr passiv verhalten, den jungen Mann selbst handeln Iassen, ihm Kranke zur Besorgung übergeben, ihn dabei anlernen, auf die noch vorhandenen Lücken seiner Kenntnisse, die sich dabei am besten ergeben, aufmerksam machen, seine Begriffe beriohtigen, und auf das Wesentliche der Praxis konzentrieren. Hierzu ist also ein gehörigcr Vorrat von Kranken erforderlich, welche von den Studierenden teils im Hospital, teils in den Häusern der Kranken besorgt werden. Dieser letzte Punkt scheint mir dazu sehr nötig, damit der junge Arzt auch alle im gewöhnlichen Leben vorkommenden Schwierigkciten der Praxis kennen und anwenden lerne.

Es folgt aus der Natur der Sache, daß ein solcher Unterricht unmöglich vielen jungen Lueuten auf einmal erteilt werden könne, weil eben hierbei das genaue Detail und das Eingehen in die individuelle Vorstellungsart und Charaktere der Zöglinge die Hauptbedingungen sind, und ich kann aus Erfahrung versichern, daß es unmöglich ist, mehr als 20 auf einmal vollkommen zu unterweisen.

Das Resultat von dem allen ist, daß dieser Teil des Unterrichts weit besser auf Akademien als in der Hauptstadt erteilt werden kann, weil hier einerseits die zu große Menge Kranken, die er anfangs auf einma sieht, ihn irre machen, anderseits der zu große Zusammenfluß der Lehrlinge jene sorgfältige und spezielle Bildung dem Lehrer unmöglich machen muß. - Hat aber der Studierende ein Jabr lang diese spezielle klinische Bildung genossen, dann wird es nützlich sein, ihn größere Hospitäler besuchen zu lassen, wo er durch die Menge Kranken jeder Art in den Stand gesetzt wird, seine praktischen Kenntnisse zu erweitern und die Natur unter den mannigfaltigsten Formen kennen zu lernen.

Es würde daher nach meiner unmaßgeblichen Meinung folgende Einrichtung des medizinischen Studiums (worunter ich immer auch die Bildung des rationellen Chirurgen verstehe) die beste sein.

1. Zwei medizinisch-chirurgische Akademien (außer der zu Berlin), welche mit hinlänglichen geschickten Lehrern und den nötigen Anstalten, d. h. Naturalienkabinett, Laboratorium, Kranken- und Gebärhaus, versehen wären.

2. Jeder junge Mensch, der zum Studio der Medizin und Chirurgie auf die Akademie gehen will, müßte erst examiniert werden, ob er die dazu nötigen Schulkenntnisse und Geistesfähigkeiten besitzt; dies ist besonders bei den studierenden Chirurgen notwendig.

3. Auf der Akademie müßte er drei Jahre zubringen und daselbst nach einem bestimmten, jedem bei seiner Inscription mitzuteilenden Plan die Vorlesungen besuchen. Alle halben Jahre würden die jungen Leute von den Lehrern, bei welchen sie Collegia gehört, examiniert. und nach dem Befund ihnen entweder erlaubt weiterzurücken oder nicht.

Die Ordnung könnte unmaßgeblich folgende sein:

I. Semestrum: Philosophie, Mathematik, Medizinische , Enzyklopädie, Anatomie (im Sommer Osteologie)

II. Semestrum: Physik, Chemie, Physiologie, Botanik.

III. Semestrum: Pathologie, Arzneimittellehre, Semiotik, Naturgeschichte, Repitition der Anatomie.

IV. Semestrum: Allgemeine Heilkunde, spezielle Heilkunde (welche ein Jahr lang geles en werden muß), Chirurgie (ebenfalls ein Jahr lang). Beide Collegia zwei Stunden täglich. 
V. Semestrum: Spezielle Heilkunde und Chirurgie fortgesetzt, Entbindungskunst, Klinik als Zuhörer.

VI. Semestrum: Klinik als Praktikant, Medizinische Litterärgeschichte, gerichtliche Medizin.

4. Nun folgten. I Jahr lang der Aufenthalt in Berlin, dessen Zweck folgender wäre:

a) Repitition der Hauptcollegien, da es in der Medizin besonders sehr nützlich ist, die Hauptlehren von verschiedenen Lehrern entwickeln zu hören, um Einseitigkeit und Nachahmung zu vermeiden.

b) Vollkommenes Studium der Anatomie mit eigener Praxis.

c) Vollkommene Ausbildung und Uebung in der medizinischen, chirurgischen und obstetrizischen Praxis in dem großen Hospital und der dazu zu errichtenden Klinik.

d) Studium der Tierarzneikunde, was auf der Akademie unmöglich und dem künftigen Kreisarzt und Wundarzt so unentbehrlich ist.

5. Hierauf der Cursus und das Examen pro Licentia praxeos.

Durch die Errichtung der chirurgischen Pepiniere ist zwar einem großen Teil der Studierenden das Triennium academicum erspart, da sie die nämlichen Collegia hier in Berlin hören, und man könnte es, was auch gewöhnlich geschieht, dafür rechnen; aber dabei fehlt der chirurgi sche Unterricht, so wie ich ihn oben geschildert habe, und so der wesentliche Teil der praktischen Bildungen. In dieser Hinsicht würde es sehr nützlieh sein, wenn auch hier in der Charité ein solches klinisches Institut errichtet würde, wozu nur 20 bis 30 Kranke aus der großen Menge ausgewählt, diese aber einer speziellen Beobachtung unterworfen an die jungen Studierenden zur Behandlung verteilt und von ihnen unter Leitung des klinischen Lehrers besorgt würden.

Auf solche Weise würde die Berliner Lehranstalt einen doppelten Zweck haben, einmal den einer ersten Bildungsakademie (in welcher Beziehung sie die dritte Akademie des preussischen Staates wäre) und zweitens den der allgemeinen und letzten Vollendung des medizinischchirurgischen Studiums für alle Aerzte der preußischen Staaten."

Allen derartigen Plänen schien durch die Niederlagen Preußens auf dem Schlachtfelde von Jena am 14. Oktober 1806 ein trostloses, unrühmliches Ende bereitet worden zu sein.

Aber die Hoffinungslosigkeit, in die auch die Besten ihrer Zeit verfielen, war nur von kurzer Dauer. Die tiefste Erniedrigung gebar die stärkste moralische Kraftentfaltung, und der glühende Drang, das geliebte Vaterland zu neuem Leben zu erwecken, spornte namentlich die Männer der Wissenschaft zu großen reformatorischen Ideen und Arbeiten an. Das Vorbild eines Fichte, eines Schleiermacher wirkte auch auf die Kleinmütigen und riß sie fort.

Die Bestrebungen zur Gründung der Berliner Universität wurden durch den unglücklichen Ausgang des Krieges unmittelbar gefördert. Die Hallenser Universität war durch $\mathrm{Na}$ poleon aufgehoben worden; sie nach Berlin zu verlegen und dort neu erstehen zu lassen, war der Entschluß, den einige ihrer Professoren alsbald faßten und zu verwirklichen suchten. Von den beiden Männern, die den Plan in einem Gesuch an den König zum Ausdruck brachten, war der eine der Jurist Schmalz, der andere der Anatom Froriep. Die am 10. August 1807 erbetene Audienz beim Könige wurde durch $\mathrm{Hufe}$ land, den königlichen Leibarzt, in Memel vermittelt und gefördert. Und indem der König sich dem Grundgedanken, eine Universität in Berlin zu errichten - wenn auch mit Rücksicht auf etwaige politische Verwicklungen nicht durch Uebernahme der Hallenser Universität - geneigt schien, wurde so unter dem bedcutsamen Anteil zweier Professoren der Medizin die lange schlummernde Saat zur ersten Entwicklung gebracht. Durch die Kabinettsordre ${ }^{1}$ ) vom 4 . September wurde Be y me beauftragt, die Einrichtung, ,einer allgemeinen Lehranstalt in Berlin in angemessener Verbindung mit der Akademie der Wissenschaften" in die Hand zu nehmen. Berufen wurde u. a. Froriep, aufgefordert zur Uebersiedlung wurde Reil, mit der Versicherung, daß, wenn er komme, man sich alle Mühe geben werde, die Klinik in der Charité nach seinen Ideen einzurichten, ferner der Anatom Loder, der früher an den Universitäten Jena und Halle gewirkt hatte, zurzeit aber als Leibarzt am Hofe zu Moskau lebte. Sowohl Reil wie Loder antworteten auf den Ruf mit begeisternder Zustimmung. Beide, ebenso $\mathrm{Hufe}$ land und Froriep reichten neben anderen Männern auf das Ersuchen Beymes neue Gutachten über die Organisation der neven Unterrichtsanstalt ein: Hufeland seine „Vorschläge

1) Ueber diese und andere Daten siehe die Festschrift von R. Köpke zur 50 jährigen Gedenkfeier für die Berliner Universität. (Berlin 1860.) zur Vereinigung des Collegii medico-chirurgici mit der allgemeinen akademischen Unterrichtsanstalt" und später "Ideen ïber die neu zu errichtende Universität zu Berlin und ihre Verbindung mit der Akademie der Wissenschaften und anderen Instituten“, Reil seinen „Entwurf zur Organisation einer wissenschaftlich medizinischen Schule".

Daß in der weiteren Entwicklung des Problems sich oft genug Schwierigkeiten in den Weg stellten, ist fast als selbstverständlich zu betrachten. Froriep wurde beim Beginn seiner Vorlesungen im Oktober 1807 durch einen Einspruch des Collegium medico-chirurgicum überrascht, das ,die Professoren des medizinischen Friedrich Wilhelm-Instituts in ihrem Vorrechte schützen wollte"; zwar wurde dieser Protest durch ein Kabinettsschreiben gegenstandslos gemacht, aber bald stellten sich neue Verwicklungen ein, die zu Frorieps frühzeitiger Amtsniederlegung führten. Auch die anderen berufenen Professoren wurden von unliebsamen Hindernissen, teils persönlicher, teils amtlicher Natur, betroffen. Trotzdem schritt, Hand in Hand mit der Vorbereitung der politischen Wiedergeburt Preußens, die Gründungsarbeit vorwärts, in erster Linie unablässig getrieben durch Fichte, zur entscheidenden Tat geführt durch Wilhelm von Humboldt, der Februar 1809 zum Leiter des Unterrichtswesens berufen wurde. Am 16. August 1809 erging die Königliche Kabinettsordre, die die Anträge Humboldts zur Verwirklichung der Universität bewilligte.

„Ich genehmige 1. die Errichtung einer Universität in Berlin und deren Verbindung mit der Akademie der Wissenschaften sowohl als der Künste, und mit den dort schon existierenden wissenschaftlichen Instituten und Sammlungen, die medizinischen mit eingeschlossen, insofern diese letzteren als rein wissenschaftlich zum akademischen Unterricht, nicht aber zu dem militairischen oder polizeylichen Medicinalwesen, oder den allgemeinen Krankenanstalten gehören ......"

In der Kommission, die die Verteilung der Räume in dem zum Universitätsgebäude bestimmten Prinz Heinrich-Palais vornehmen sollte, saß auch der Geheime Obermedizinalrat Kohlrausch. Das Laboratorium für Anatomie wurde in das sog. Stallgebäude verlegt, im zweiten Stockwerk wurden fünf Zimmer und die große Galerie dem anatomischen Museum, im dritten Stockwerk ein Zimmer für das Bandagenkabinett eingeräumt.

Anfang 1810 wurde Reil zum Professor der Therapie und Direktor der wissenschaftlichen Deputation, der Anatom Rudolphi aus Greifswald, der Physiologe Horkel aus Halle berufen.

Am 7. Mai begann Hufeland als erster medizinischer Professor an der Universität seine Vorlesungen, und zwar ein Privatkolleg über praktische Medizin und ein Publicum über Makrobiotik

Am 23. Mai 1810 beantragte v. Humboldt in seinem an den König erstatteten ,Generalbericht über die Begründung der Universität" deren Eröffinung zu "Michael" $1810 . \mathrm{Zu}$ diesem Zweck erbat er die Vollmacht, in der Organisation der Universität mit Berufung neuer Lehrer und Einrichtung der notwendigen Anstalten weiter vorzugehen; und zwar motivierte er seine Forderungen für die Medizin mit folgenden Sätzen :

„Die medicinische Facultät ist diejenige, welche hier am leichtesten zu einem gewissen Grade der Vollständigkeit gebracht werden kann, und es ist daher nothwendig, auch ihr zuerst die meiste Aufmerksamkeit zu widmen.

Das Wichtigste zur Bildung angehender Aerzte ist nun die An. legung von Krankenanstalten, in welchen dieselben zur wissenschaftlichen Kenntniss und Heilung der Krankheiten praktische Anleitung erhalten. In ihrem vollständigen Zustand würde die Universität deren viere, nemlich ein medicinisches, ein chirurgisches, eins für Gemüthskranke, eins für Gebährerinnen bedürfen. Jetzt aber können die beiden ersteren genügen. Die Charité taugt zu diesem Behufe nicht. Die Menge der Kranken zerstreut den Anfänger, und macht es dem Lehrer unmöglich bei einem Einzelnen gehörig zu verweilen." Wollte man aber eine kleine Zahl im Gebäude selbst abšondern, so würden dadurch die Kosten nicht viel geringer, als in einem eignen Local werden, auch, da die Kranken, an welchen der Studierende geübt werden soll, alle mögliche körperliche Pflege und Sorgfalt geniessen, und daher weit besser gehalten werden müssen, als es in einem Hospital möglich ist, eine ungleiche Wirtbeshaft und eine Menge von Collisionen entstehen. Endlich ist die Charité zu weit von der Stadt entfernt. 
Es wird daher nothwendig, zwei kleine Krankenhäuser, ein medicinisches und ein chirurgisches, jedes zu 12 Betten anzulegen. Das erste würde dem Oberbergrath Reil. das letzte dem noch zu berufenden Professor der Chirurgie übergeben. Jedes würde, wie auch das Königsbergische und zwei in Halle ausgestattet sind, jährlich 3000 Reichsth. brauchen, die von dem Januar c. an bezahlt, zugleich zu den Kosten der ersten Einrichtung und der Anschaffung der Ưtensilien hinreichen würden.

Das Local für diese Anstalten müßte für jetzt nur gemiethet werden, künftig aber würde, da dieselben auch gleich im ersten Bau eine eigene Einrichtung erfordern, es nothwendig seyn eigene Gebäude für dieselben, dicht bei einander, aufzuführen, wozu auch der von E. K. M. Allerhöchster Gnade den hiesigen wissenschaftlichen Anstalten bestimmte Fonds hinreichen wird."

Eine Ergänzung der ordentlichen medizinischen Professoren wurde durch Obermedizinalrat Knape vom Friedrich WilhelmsInstitut für Anatomie und durch Grae fe für Chirurgie erreicht; als außerordentlicher Professor wurde der frühere Militärarzt Reich (bekannt durch seine "Fieberlehre") angestellt, zum Beitritt als Privatdozent wurde $\mathrm{N}$ as se aus Elberfeld aufgefordert; freiwillig trat Horn, der Dirigent des Klinikums in der Charité, und Obermedizinalrat Kohlrausch dem Lehrkörper bei. Nach dem am 22. September dem König überreichten Schlußbericht entfielen unter den 58 Dozenten auf die medizinische Fakultät sechs Ordinarien (Hufeland, Graefe, Horkel, Knape, Reil, Rudolphi), ein Extraordinarius (Reich), sieben Privatdozenten (Bernstein, Friedländer, Horn, Kohlrausch, Beckleben, Staberoh, Wolfart). Diese kündigten insgesamt 34 Vorlesungen an, und zwar Enzyklopädie der Arzneiwissenschaft (Rudolphi), Geschichte derselben (Reich), Physiologie (Horkel und Rudolphi), Psychologie (Reil), vergleichende und pathologische Anatomie (Rudolphi), Osteologie, Syndesmologie und Splanchnologie (Knape), Pathologie (Reil), Fieberlehre (Reich und Horn), Materia medica (Hufeland, Friedländer, Staberoh), praktische Heilkunde (Hufeland), Entbindungskunst (Friedländer, Kohlrausch), Chirurgie (Graefe), Acologie (Bernstein), Semiotik und Formellehre (Wolfart), Medicina forensıs (Knape), klinische Uebungen ( $\mathrm{Hufeland,} \mathrm{Graefe,}$ Knape, Reil, Horn, Kohlrausch), Tierarzneikunde (Reckleben).

Der Bericht wurde am 28. September vom Könige genehmigt und gleichzeitig der Rektor und die vier Dekane ernannt, für die Medizinische Fakultät $\mathrm{Hufeland.}$

Am 6. Oktober wurde die erste Immatrikulation von Studenten vorgenommen; unter den sechs Studierenden war ein Mediziner, nämlich August Wilhelm Heim aus Berlin.

Am 10. Oktober 4 Uhr versammelten sich auf Einladung des Rektors die Dekane und mehrere ordentliche Professoren zum ersten Male im Universitätsgebäude. Der Rektor eröffnete die Versammlung mit einer Anrede; er nahm die Professoren durch Handschlag an Eidesstatt in Pflicht, dem Könige treu und gehorsam zu sein und dem Staat und der Universität sich ganz zu widmen. Darauf wurde der Senat der Universität für konstituiert erklärt und ein Senatsreglement angenommen. Die Vorlesungen sollten erst Ende Oktober begonnen werden. In der Medizinischen Fakultät eröffnete Hufeland am 15. Oktober seine Vorlesungen und Uebungen im Poliklinischen Institut, dessen Abteilungen von Bernstein und Flemming geleitet wurden, ebenso Graefe und Klaproth.

Die Pforten der neuen Universität waren geöffnet, von der Clemens Brentano in seinem zu ihrer (ganz prunklosen) Einweihung verfaßten Gedichte sang:

\footnotetext{
„Glück auf, Glück auf! Viktoria!

Es ist im Vaterlande

Ein Musenberg voll Gloria

Mit Gottes Gunst entstanden."
}

Nun, wo alle die Hoffinungen, die man an ihre Gründung geknüpft hat, durch ein Säkulum in überreichem Maße erfüllt worden sind, können wir mit Genugtuung der Mitarbeit gedenken, die auch hervorragende Männer der Heilkunde bei der Schöpfung dieses segensreichen Werkes geleistet haben. 